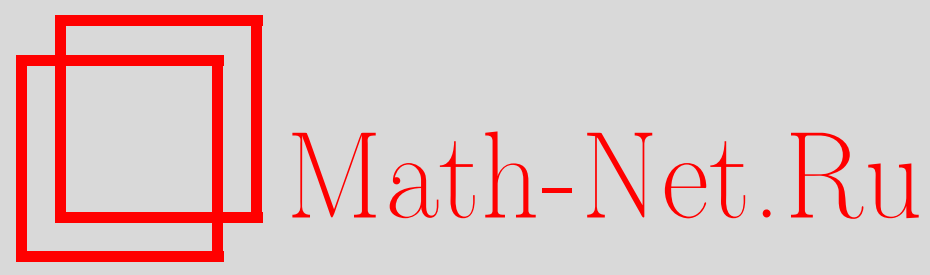

Н. Г. Мощевитин, Наилучшие совместные приближения: нормы, сигнатуры и асимптотические направления, $M a$ тем. заметки, 2000, том 67, выпуск 5, 730-737

DOI: https://doi.org/10.4213/mzm890

Использование Общероссийского математического портала Math-Net.Ru подразумевает, что вы прочитали и согласны с пользовательским соглашением http://www . mathnet.ru/rus/agreement

Параметры загрузки:

IP : 54.224 .135 .184

26 апреля 2023 г., $15: 33: 41$

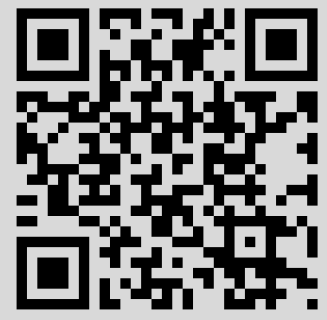


УДК 511

\section{НАИЛУЧШИЕ СОВМЕСТНЫЕ ПРИБЛИЖЕНИЯ: НОРМЫ, СИГНАТУРЫ И АСИМПТОТИЧЕСКИЕ НАПРАВЛЕНИЯ}

\section{Н. Г. Мощевитин}

Получены результаты о распределении направлений наилучших совместных приближений в различных нормах.

Библиографой: 17 названий.

1. Обозначения. Под выпуклой симметричной лучевой функиией в евклидовом пространстве $\mathbb{R}^{n}$ со стандартньм скалярным произведением понимается функция $f: \mathbb{R}^{n} \rightarrow \mathbb{R}_{+}$со свойствами:

1) $f$ непрерывна;

2) $f(x) \geqslant 0 \forall x \in \mathbb{R}^{n}, f(x)=0 \Longleftrightarrow x=0$;

3) $f(-x)=f(x) \forall x \in \mathbb{R}^{n}$;

4) $f(t x)=t f(x) \forall x \in \mathbb{R}^{n}, \forall t \in \mathbb{R}_{+}$;

5) множество $B_{f}^{1}=\left\{y \in \mathbb{R}^{n}: f(y) \leqslant 1\right\}$ выпукло и $0 \in \operatorname{int} B_{f}^{1}$.

Согласно [1] в таком случае $f$ задает какую-то другую норму в $\mathbb{R}^{n}$. Функция (или норма) $f$ называется строго выпуклой, если множество $B_{f}^{1}$ является строго выпуклым, т.е. его граница $\partial B_{f}^{1}$ не содержит прямолинейных отрезков. Через $B_{f}^{\lambda}(a)$ будем обозначать множество

$$
B_{f}^{\lambda}(a)=\left\{y \in \mathbb{R}^{n}: f(y-a) \leqslant \lambda\right\},
$$

так что $B_{f}^{1}=B_{f}^{1}(0)$.

Для вектора $\alpha=\left(\alpha_{1}, \ldots, \alpha_{n}\right) \in \mathbb{R}^{n}$ определим $f$-наилучшее совместное приближение (сокращенно $f$-н.п.) как целую точку $\tau=\left(p, a_{1}, \ldots, a_{n}\right) \in \mathbb{Z}^{n+1}$ такую, что $p \geqslant 1$ и $f(\alpha q-b)>f(\alpha p-a)$ для всех $\left(q, b_{1}, \ldots, b_{n}\right) \in \mathbb{Z}^{n+1}, 1 \leqslant q \leqslant p-1$, и для всех $\left(p, b_{1}, \ldots, b_{n}\right) \in \mathbb{Z}^{n+1}, b \neq a$. В случае, когда $f$ такова, что $B_{f}^{1}=\left\{y=\left(y_{1}, \ldots, y_{n}\right) \in \mathbb{R}^{n}\right.$ : $\left.\max _{j}\left|y_{j}\right| \leqslant 1\right\}$, наше определение задает обычные наилучшие совместные приближения.

Все $f$-н.п. к набору $\alpha$ располагаются в виде последовательностей

$$
\begin{gathered}
\tau_{\nu}=\left(p_{\nu}, a_{\nu}\right) \in \mathbb{Z}^{n+1}, \quad p_{\nu} \in \mathbb{N}, \quad a_{\nu}=\left(a_{1, \nu}, \ldots, a_{n, \nu}\right) \in \mathbb{Z}^{n}, \\
p_{1}<p_{2}<\cdots<p_{\nu}<\cdots, \\
f\left(\alpha p_{1}-a_{1}\right)>f\left(\alpha p_{2}-a_{2}\right)>\cdots>f\left(\alpha p_{\nu}-a_{\nu}\right)>\cdots,
\end{gathered}
$$

причем эти последовательности являются конечньми или бесконечными в зависимости от того, имеет место принадлежность $\alpha \in \mathbb{Q}^{n}$ или нет.

Работа выполнена при финансовой поддержке Российского фонда фундаментальных исследований, гранты № 99-01-00357 и № 00-15-96109. 
Через $\xi_{\nu}=\left(\xi_{1, \nu}, \ldots, \xi_{n, \nu}\right)$ будем обозначать вектор отклонений $\xi_{j, \nu}=\alpha_{j} p_{\nu}-a_{j, \nu}$. Положим $\Xi_{\nu}=\left(\Xi_{1, \nu}, \ldots, \Xi_{n, \nu}\right), \Xi_{j, \nu}=\xi_{j, \nu} / f\left(\xi_{\nu}\right)$, так что $\Xi_{\nu} \in B_{f}^{1}$. Для произвольного вектора $\xi \in \mathbb{R}^{n}, \xi \neq 0$, также будем использовать обозначение $\Xi(\xi)=\xi / f(\xi) \in B_{f}^{1}$. Кроме того, для целочисленного вектора $\zeta=\left(p, a_{1}, \ldots, a_{n}\right) \in \mathbb{R}^{n+1}$ будем пользоваться обозначением $\xi^{\alpha}(\zeta)=\left(p \alpha_{1}-a_{1}, \ldots, p \alpha_{n}-a_{n}\right) \in \mathbb{R}^{n}$.

В настоящей работе мы устанавливаем несколько простых общих фактов о поведении величин $\Xi_{\nu}$. Но преж де всего мы приведем некоторые простые результаты, касающиеся порядка наилучших приближений.

2. Порядок наилучших приближений. Из теоремы Минковского о вьпуклом теле, примененной к цилиндру

$$
\Omega_{\nu}=\left\{z=\left(x, y_{1}, \ldots, y_{n}\right) \in \mathbb{R}^{n+1}:|x|<p_{\nu+1}, f(\alpha x-y)<f\left(\xi_{\nu}\right)\right\}
$$

следует, что для всякого $\nu$ вьполнено

$$
f\left(\xi_{\nu}\right) \leqslant C_{1}(f) p_{\nu+1}^{-1 / n}
$$

с константой $C_{1}(f)=2 /\left(\mathrm{Vol} B_{f}^{1}\right)^{1 / n}$. С другой стороны, мы отметим

ПРЕДЛОЖЕНИЕ 1. Если $\operatorname{dim}_{\mathbb{Z}}\left(1, \alpha_{1}, \ldots, \alpha_{n}\right) \geqslant 3, m o$

$$
f\left(\xi_{\nu}\right) p_{\nu+1} \rightarrow+\infty, \quad \nu \rightarrow+\infty
$$

ДокАЗАТЕЛьСтво. 1) Пусть $\Lambda^{2} \in \mathbb{Z}^{s+1}$ - произвольная двумерная подрешетка и $\operatorname{det}_{2} \Lambda^{2}$-ее двумерньй фундаментальньй объем. Тог да множество решеток $\left\{\Lambda^{2} \subset \mathbb{Z}^{s+1}\right.$ : $\left.\operatorname{det}_{2} \Lambda^{2} \leqslant \gamma\right\}$ конечно для любого положительного $\gamma$.

2) Рассмотрим двумерную решетку $\Lambda_{\nu}^{2}=\left\langle\tau_{\nu}, \tau_{\nu+1}\right\rangle_{\mathbb{Z}}$. Из условия $\operatorname{conv}\left(0, \tau_{\nu}, \tau_{\nu+1}\right)$ $\subset \Omega_{\nu}$ следует

$$
\frac{1}{2} \operatorname{det}_{2} \Lambda_{\nu}^{2}=\operatorname{vol}_{2}\left(\operatorname{conv}\left(0, \tau_{\nu}, \tau_{\nu+1}\right)\right) \ll f\left(\xi_{\nu}\right) p_{\nu+1}
$$

3) Из условия $\operatorname{dim}_{\mathbb{Z}}\left(1, \alpha_{1}, \ldots, \alpha_{s}\right) \geqslant 3$ следует (см. [2, утверджение 2]), что последовательность наилучших приближений не лежит асимптотически ни на какой двумерной решетке, и легко видеть, что ни при каком конечном $\nu_{0}$ не может выполняться

$$
\bigcup_{\nu=1}^{\infty} \tau_{\nu} \subset \bigcup_{n=1}^{\nu_{0}} \Lambda_{\nu}^{2} .
$$

Предложение 1 вытекает из 1)-3).

Следующее утверждение (фактически доказанное Хинчиным [3], [4]) показывает, что в соотношении (3) никакой определенной скорости стремления к бесконечности величины $f\left(\xi_{\nu}\right) p_{\nu+1}$ установить нельзя.

ПРЕДЛОЖЕНИЕ 2. ДЛя произвольной функиии $\psi(y) \uparrow+\infty, y \rightarrow+\infty$, сколь угодно медленно монотонно возрастающей $\kappa$ бескончности, найдется набор $\alpha \in \mathbb{R}^{n}$, $\operatorname{dim}_{\mathbb{Z}}\left(1, \alpha_{1}, \ldots, \alpha_{n}\right)=n+1\left(\alpha_{1}, \ldots, \alpha_{s}\right.$ линейно независимы вместе $с$ единицей над $\mathbb{Z})$ такой, что

$$
f\left(\xi_{\nu}\right) p_{\nu+1}=O\left(\psi\left(p_{\nu+1}\right)\right), \quad \nu \rightarrow+\infty
$$


Соотношение (4) показывает, что при $n \geqslant 2$ найдутся наборы $\alpha$, для которых из оценок $(2),(3)$ точной будет являться нижняя оценка. Подобного рода результаты связаны с наличием вырождений. Отметим, что факт вырождения размерности отмечался в [5], [2]. Вырождения для задачи о линейных формах (на самом деле связанные с сингулярными системами Хинчина [3], [4]) рассматривались автором в [6], в [7] имеется обзор близких результатов и приложений. Результаты об общих законах апшроксимации имеются в [8].

При $n=1$ предложение 1 не имеет места и для всех $\nu$ вьполнено

$$
C_{2}(f) p_{\nu+1}^{-1} \leqslant f\left(\xi_{\nu}\right) \leqslant C_{1}(f) p_{\nu+1}^{-1}
$$

(см. [9]).

\section{3. Последовательные приближения.}

Tеорема 1. Выполнено $\Xi_{\nu+1} \notin \operatorname{int} B_{f}^{1}\left(\Xi_{\nu}\right) \quad \forall \nu \in \mathbb{N}$.

Теорема 1 обобщает теорему Роджерса о сигнатурах для обычных н.п. из [10].

ДокАЗАТЕльство. По определению $f$-н.п. в цилиндре $\Omega_{\nu}($ см. $(1))$ ненулевых целых точек нет. Точка

$$
\tau_{\nu+1}-\tau_{\nu}=\left(p_{\nu+1}-p_{\nu}, a_{1, \nu+1}-a_{1, \nu}, \ldots, a_{n, \nu+1}-a_{n, \nu}\right)
$$

лежит вне цилиндра $\Omega_{\nu}$, но $0<p_{\nu+1}-p_{\nu}<p_{\nu+1}$, так что условие $\tau_{\nu+1}-\tau_{\nu} \notin \Omega$ равносильно тому, что

$$
\xi_{\nu+1} \notin \operatorname{int} B_{f}^{f\left(\xi_{\nu}\right)}\left(\xi_{\nu}\right)
$$

(надо рассмотреть параллельную проекцию цилиндра $\Omega$ на плоскость координат $y_{1}, \ldots$, $y_{n}$ вдоль его оси). Замечая, что $0 \in \partial S_{f}^{f\left(\xi_{\nu}\right)}\left(\xi_{\nu}\right)$, в силу вьпуклости видим, что

$$
\xi_{\nu+1} \frac{f\left(\xi_{\nu}\right)}{f\left(\xi_{\nu+1}\right)} \notin \operatorname{int} B_{f}^{f\left(\xi_{\nu}\right)}\left(\xi_{\nu}\right) .
$$

Последнее соотношение равносильно утерждению теоремы 1.

Теорема 1 доказана.

Отметим, что соотношение (5) несколько более общее, чем заключение теоремы 1.

\section{4. Строго выпуклые нормы.}

Теорема 2. Пусть норма $f$ строго выпукла. Тогда найдется $\delta=\delta(f)>0$ такое, что для каждого набора $\alpha \notin \mathbb{Q}^{n}$ найдется бесконечно много у таких, что $\Xi_{\nu+1} \notin B_{f}^{1+\delta}\left(\Xi_{\nu}\right)$.

Напомним, что набор $\alpha=\left(\alpha_{1}, \ldots, \alpha_{n}\right)$ назыается плохо приближаемым, если с некоторой постоянной $D(\alpha)>0$ вьполнено неравенство

$$
\max _{1 \leqslant j \leqslant n} \min _{a_{j} \in \mathbb{Z}}\left|p \alpha_{j}-a_{j}\right| \geqslant D(\alpha) p^{-1 / n} \quad \forall p \in \mathbb{N} .
$$

(О сушествовании такого рода наборов см. [8], [11], [12].) Легко видеть, что набор $\alpha$ плохо приближаем тогда и только тогда, когда для каждой нормы $f$ выполнено

$$
\min _{a \in \mathbb{Z}^{n}} f(p \alpha-a) \geqslant D_{1}(f, \alpha) p^{-1 / n} \quad \forall p \in \mathbb{N} .
$$


ТЕорема 3. Пусть набор а плохо приближаем и норма $f$ строго выпукла. Тогда найдутся постоянные $w=w(D, f) \in \mathbb{N} u \delta=\delta(D, f)>0$, обладаюшие следуюшим свойством: для любого $\nu \geqslant 1$ найдется натуральное $j$ из интервала $\nu \leqslant j \leqslant \nu+w$, дя которого выполнено

$$
\Xi_{j+1} \notin B_{f}^{1+\delta}\left(\Xi_{j}\right)
$$

Теорема 2 показывает, что для строго вьпуклой нормы условие $\theta_{\nu+1} \notin \operatorname{int} B_{f}^{1}(\theta)$ для последовательности $\theta_{\nu} \in B_{f}^{1}$, вообще говоря, не является достаточным для существования $\alpha$ такого, что

$$
\lim _{\nu \rightarrow \infty}\left(\theta_{\nu}-\Xi_{\nu}\right)=0 .
$$

Теорема 3 утверждает , что для плохо приближаемых наборов чисел те $j$, для которых выполнено (7), появляются регулярно. По-видимому, результат теоремы 3 не связан со свойством плохо приближаемости набора $\alpha$, и справедлива более сильная форма теоремы 2 , но в настоящей работе это не доказано. Доказательство теорем 2,3 проведено в п. 5. Из результатов п. 6 следует, что теорема 2 становится неверной для нестрого выпуклых норм.

5. Две леммы. В [13] доказано следующее утверждение.

ЛЕмма 1. Для всякой нормы $f$ с постонной $h=2^{n+1}$ выполнено $p_{\nu+h} \geqslant 2 p_{\nu}$ $\forall \nu$.

СлЕДСТВИЕ 1. Для каждого набора $\alpha \notin \mathbb{Q}^{n}$ для всех $\nu, j>1$ выполнено

$$
f\left(\xi_{\nu+j h}\right) \leqslant C_{1} p_{\nu}^{-1 / n}\left(\frac{1}{2}\right)^{j / n}
$$

Следствие 1 очевидно, и мы его не доказьваем.

СлЕДСТВИЕ 2. Если набор а плохо приближаем, то найдется постоянная $h^{*}=$ $h^{*}(f, \alpha) \in \mathbb{N}$ такая, что

$$
f\left(\xi_{\nu+h^{*}}\right)<\frac{1}{2} f\left(\xi_{\nu}\right) \quad \forall \nu \geqslant 1
$$

ДоКАЗАТЕЛЬСТВо. Из (2) и условия плохо приближаемости (6) следует

$$
D_{1} p_{\nu}^{-1 / n} \leqslant f\left(\xi_{\nu}\right) \leqslant C_{1} p_{\nu}^{-1 / n}
$$

а по лемме $1 p_{\nu}$ экспоненциально возрастают. Из сказанного выше вытекает соотношение (8).

Лемма 2. Пусть норма $f$ строго выпукла. Тогда для всякого $\varepsilon>0$ найдется $\delta>0$ такое, что для любого $\theta \in \partial B_{f}^{1}(0)$ и для любого $\xi \in B_{f}^{1}(0) \backslash B_{f}^{1}(\theta)$ такого, что

$$
\Xi(\xi) \in \partial B_{f}^{1}(0) \cap\left(B_{f}^{1+\delta}(\theta) \backslash B_{f}^{1}(\theta)\right),
$$

выполнено $f(\xi)>1-\varepsilon$.

ДокАЗАТЕЛЬСТВо. Пусть $\eta \in \partial B_{f}^{1}(\theta) \cap \partial B_{f}^{1}(0)$. В силу строгой выпуклости интервал $(0 ; \eta) \subset \operatorname{int} B_{f}^{1}(\theta)$. Если $\Xi \in \partial B_{f}^{1}(0) B_{f}^{1}(\theta)$ лежит в малой $\delta$-окрестности точки $\eta$, то по непрерывности отрезок $[0 ; \Xi]$ пересекается с $\partial B_{f}^{1}(\theta)$ в некоторой точке $\zeta(\Xi)=[0 ; \Xi] \cap$ $\left(\partial B_{f}^{1}(\theta) \backslash 0\right)$, причем $\zeta(\Xi) \rightarrow \eta$ при $\Xi \rightarrow \eta$. Ясно, что если $\xi \in B_{f}^{1}(0) B_{f}^{1}(\theta)$, то $\xi$ лежит между $\Xi(\xi)$ и $\zeta(\Xi(\xi))$, и лемма доказана. 
6. Доказательство теорем 2 и 3. Докажем теорему 2.

Если утверждение теоремы 2 не выполнено, то для любого $\delta>0$

$$
\Xi_{\nu+1} \in B_{f}^{1+\delta}\left(\Xi_{\nu}\right) \quad \text { при } \nu \geqslant \nu_{0}(\delta) .
$$

Теперь из теоремы 1 и леммы 2 следует, что для любого $\varepsilon>0$

$$
f\left(\xi_{\nu+1}\right) \geqslant(1-\varepsilon) f\left(\xi_{\nu}\right) \quad \text { при } \nu \geqslant \nu_{0}(\varepsilon),
$$

т.e.

$$
f\left(\xi_{\nu_{0}+j}\right) \geqslant(1-\varepsilon)^{j} f\left(\xi_{\nu_{0}}\right) .
$$

Но из следствия 1 леммы 1 имеем

$$
f\left(\xi_{\nu_{0}+j}\right) \leqslant C_{1} p_{\nu_{0}}^{-1 / n}\left(\frac{1}{2}\right)^{j / n} .
$$

При достаточно малом $\varepsilon$ неравенства (9) и (10) противоречивы при $j \rightarrow \infty$.

Теорема 2 доказана.

Теперь докажем теорему 3.

Пусть утверждение теоремы 3 не вьполнено. Тогда для сколь угодно большого $w \in \mathbb{N}$ и сколь угодно малого $\delta>0$ найдется $\nu$ такое, что

$$
\Xi_{j+1} \in B_{f}^{1+\delta}\left(\Xi_{j}\right), \quad j=\nu, \nu+1, \ldots, \nu+w .
$$

В силу леммы 2 это означает, что

$$
f\left(\xi_{\nu+w}\right) \geqslant(1-\varepsilon)^{w} f\left(\xi_{\nu}\right),
$$

причем $\varepsilon$ может быть взято сколь угодно малым. В то же время из следстия 2 леммы 1 вытекает неравенство

$$
f\left(\xi_{\nu+w}\right) \leqslant\left(\frac{1}{2}\right)^{\left[w / h^{*}\right]} f\left(\xi_{\nu}\right) .
$$

При достаточно малом $\varepsilon$ неравенства (11) и (12) противоречивы при $w \rightarrow \infty$.

Теорема 3 доказана.

7. Теорема о сигнатуре и ее обобщение. Сигнатурой вектора $\eta=\left(\eta_{1}, \ldots, \eta_{n}\right)$ назьвается набор $\operatorname{sign} \eta=\left(\operatorname{sign} \eta_{1}, \ldots, \operatorname{sign} \eta_{n}\right)$. Роджерс [10] показал что для обычных н.п. (соответствующих случаю $B_{f}^{1}=\left\{y: \max _{j}\left|y_{j}\right| \leqslant 1\right\}$ ) последовательные н.п. удовлетворяют условию $\operatorname{sign} \xi_{\nu} \neq \operatorname{sign} \xi_{\nu+1} \forall \nu$. (Этот результат обобщается нашей теоремой 1 из п. 2.) С другой стороны, в [14] показано, что для любой наперед заданной последовательности сигнатур $\left\{\sigma_{\nu}\right\}$ такой, что $\sigma_{\nu} \neq \sigma_{\nu+1}$, найдется набор $\alpha \in \mathbb{R}^{n}$ вещественных чисел $\alpha_{1}, \ldots, \alpha_{n}$ линейно независимых вместе с 1 над $\mathbb{Z}$ таких, что sign $\xi_{\nu}=\sigma_{\nu}$. Ниже мы обобщаем этот результат.

Пусть имеется последовательность $\theta_{\nu} \in \partial B_{f}^{1}(0)$. Определим два условия.

УСловие А. Вьполнено $-\theta_{\nu+1} \in \operatorname{int} B_{f}^{1}\left(\theta_{\nu}\right) \forall \nu$.

Пусть норма $f$ гладкая (т.е. в каждой точке сфера $\partial B_{f}^{1}$ имеет единственную касательную гиперплоскость). Для точки $\theta \in \partial B_{f}^{1}(0)$ определим множество $G(\theta) \subset \partial B_{f}^{1}(0)$ следуюшим образом. Рассмотрим касательную гиперплоскость $\pi$ для множества $\partial B_{f}^{1}(\theta)$ в точке $0 \in \partial B_{f}^{1}(\theta)$. Через П обозначим то замкнутое полупространство в $\mathbb{R}^{n}$ с границей $\pi$, которое не содержит точку $\theta$. Определим теперь $G(\theta)=\Pi \cap \partial B_{f}^{1}$. 
УСловие Б. Норма $f$ гладкая и $\theta_{\nu+1} \in G\left(\theta_{\nu}\right) \forall \nu$.

Теорема 4. Пусть норма $f$ и последовательность $\theta_{\nu}$ таковы, что выполнено условие А или условие Б. Тогда существует набор $\alpha=\left(\alpha_{1}, \ldots, \alpha_{n}\right)$ чисел, линейно независимых вместе $с$ единичей над $\mathbb{Z}$, такой, что

$$
\lim _{\nu \rightarrow+\infty}\left|\Xi_{\nu}-\theta_{\nu}\right|=0
$$

ЗАмечАниЕ. В формуле (13) стремление к нулю может быть сколь угодно быстрым.

При $s=2$ рассмотрим норму $f^{*}\left(x_{1}, x_{2}\right)$, единичный шар которой задается неравенствами $\left|x_{1}+x_{2}\right| \leqslant 4,\left|x_{1}-x_{2}\right| \leqslant 1$. Применение теоремы 4 с учетом простой геометрии взаимного расположения шаров $B_{f}^{1}(0), B_{f}^{1}(\theta)$ влечет следующее утверждение.

Теорема 5. Для нормы $f^{*}(x)$ последовательность $f$-н.n. может иметь постоянную последовательность сигнатур $\sigma_{\nu}=(+,+) \forall \nu$.

Теорема 5 показьвает, что цитировавшаяся выше теорема Роджерса [10] для нормы $f^{*}(x)$ становится неверной. Ясно, что имеет место аналогичный многомерный пример и что можно рассмотреть пример со строго выпуклой нормой.

Особо отметим, что автору не удалось привести пример нормы $f$, в которой последовательность $f$-н.п. может иметь совершенно любую заданную наперед последовательность сигнатур. Автору кажется, что подобным свойством обладает евклидова норма $f\left(x_{1}, \ldots, x_{n}\right)=\sqrt{x_{1}^{2}+\cdots+x_{n}^{2}}$, однако это лишь предположение.

Технически доказательство теоремы 4 громоздко, и мы приведем его схематически.

СХЕМА ДОКАЗАТЕЛЬСТВА ТЕОРЕМЫ 4 . Предположим, что вьПолнено условие А.

Точно так, как и в работе [2], с помошью некоторого индуктивного процесса строится последовательность целых точек $\tau_{\nu}=\left(p_{\nu}, a_{1, \nu}, \ldots, a_{n, \nu}\right)$, которые и будут образовывать последовательность $f$-н.п. для предельной точки $\lim _{\nu \rightarrow+\infty}\left(a_{1, \nu} / p_{\nu}, \ldots, a_{n, \nu} / p_{\nu}\right)$.

Основание индуктивного процесса очевидно. Опишем его шаг. Пусть мы уже построили целые точки $\tau_{1}, \ldots, \tau_{\nu} \in \mathbb{Z}^{n+1}, \tau_{j}=\left(p_{j} ; a_{j}\right)=\left(p_{j}, a_{1, j}, \ldots, a_{n, j}\right), 1 \leqslant p_{1}<p_{2}<$ $\cdots<p_{\nu}$, с условиями:

1) $\tau_{1}, \ldots, \tau_{\nu}$ есть набор всех $f$-н.п. к рациональному вектору $\beta^{\nu}=\left(a_{1, \nu} / p_{\nu}, \ldots\right.$, $\left.a_{n, \nu} / p_{\nu}\right)$

2) $\Xi\left(\xi^{\beta^{\nu}}\left(\tau_{j}\right)\right)-\theta_{j}$ мало для всех $j=1, \ldots, \nu-1$;

3) $-\theta_{j} \in \operatorname{int} B_{f}^{1}\left(\Xi\left(\xi^{\beta^{\nu}}\left(\tau_{j-1}\right)\right)\right)$ при всех $j=1, \ldots, \nu$.

Покажем, как теперь определить целую точку $\tau_{\nu+1}=\left(p_{\nu+1} ; a_{\nu+1}\right)=\left(p_{\nu+1}, a_{1, \nu+1}\right.$, $\left.\ldots, a_{n, \nu+1}\right), p_{\nu}<p_{\nu+1}$, так, чтобы

$\left.1^{*}\right) \tau_{1}, \ldots, \tau_{\nu+1}$ были бы $в$ се $f$-н.п. к рациональному вектору $\beta^{\nu+1}=\left(a_{1, \nu+1} / p_{\nu+1}\right.$, $\left.\ldots, a_{n, \nu+1} / p_{\nu+1}\right)$

$\left.2^{*}\right) \Xi\left(\xi^{\beta^{\nu+1}}\left(\tau_{j}\right)\right)-\theta_{j}$ было бы мало для всех $j=1, \ldots, \nu$;

$\left.3^{*}\right)-\theta_{j} \in \operatorname{int} B_{f}^{1}\left(\Xi\left(\xi^{\beta^{\nu+1}}\left(\tau_{j-1}\right)\right)\right)$ при всех $j=1, \ldots, \nu+1$.

Рассмотрим малую окрестность $B_{f}^{\lambda}\left(a_{\nu}\right)$. Если $\lambda$ достаточно мало, то для любого $\beta \in B_{f}^{\lambda}\left(a_{\nu}\right)$ целые точки $\tau_{1}, \ldots, \tau_{\nu-1}$ будут представлять собой все первые последовательные $\nu-1 f$-н.п. к вектору $\beta$. Трудность заключается в том, что при любом $\lambda$ для некоторых $\beta \in B_{f}^{\lambda}\left(a_{\nu}\right)$ между $\tau_{\nu-1}$ и $\tau_{\nu}$ может появиться новое $f$-н.п., которое надо проконтролировать.

Рассмотрим шар $B_{f}^{1}$ и точку $\Xi\left(\xi^{\beta^{\nu}}\left(\tau_{\nu-1}\right)\right) \in \partial B_{f}^{1}$. Пусть $B^{*}=B_{f}^{1}\left(\Xi\left(\xi^{\beta^{\nu}}\left(\tau_{\nu-1}\right)\right)\right)$. Из индуктивного условия 3$)$ имеем $-\theta_{\nu} \in \operatorname{int} B^{*}$. 
Рядом с точкой $-\theta_{\nu}$ во множестве $\partial B_{f}^{1} \cap$ int $B^{*}$ найдется точка $\Xi^{*}$ такая, что двумерная плоскость $\pi^{*}$, проходящая через точки $0, \tau_{\nu}, \zeta^{*}, \zeta^{*}=\left(p_{\nu}, \Xi^{*}\right)$, будет вполне рациональна, сама точка $\Xi^{*}$ будет мало отличаться от точки $-\theta_{\nu}$ и по непрерьвности можно добиться вьполнения принадлежности $-\theta_{\nu+1} \in \operatorname{int} B_{f}^{1}\left(\Xi^{*}\right)$ (используем условие A).

Вовполне рациональной плоскости $\pi^{*}$ выбирается далекая целая точка $\tau_{\nu+1}=\left(p_{\nu+1}\right.$; $a_{\nu+1}$ ), расположенная так, что $\tau_{\nu+1}$ и $\zeta^{*}$ лежат по одну сторону от прямой $0 \tau_{\nu}$ (плоскость $\pi^{*}$ двумерна). Поскольку $\Xi^{*} \in \operatorname{int} B^{*},\left(\right.$ используем вьпуклость $B^{*}$ ) получаем, что полуоткрытый интервал $\left(0 ; \Xi^{*}\right)$ содержится во внутренности int $B^{*}$. Теперь выбор точки $\tau_{\nu+1}$ очень близко к прямой $0 \tau_{\nu}$ обеспечивает, что $\tau_{1}, \ldots, \tau_{\nu+1}$ будут именно все $f$-н.п. к рациональному вектору $\beta^{\nu+1}=\left(a_{1, \nu+1} / p_{\nu+1}, \ldots, a_{n, \nu+1} / p_{\nu+1}\right)$.

Сходимость последовательности векторов $\beta^{\nu}$ обеспечивается малым отличием вектора $\beta^{\nu+1}$ от $\beta^{\nu}$.

Линейная независимость над $\mathbb{Z}$ предельных чисел $1, \alpha_{1}, \ldots, \alpha_{n}$ обеспечивается так же, как и в работе [2], “подкручиванием".

Шаг индуктивного процесса описан.

При условии А утверждение теоремы 4 будет выполняться для построеных в результате индуктивного процесса чисел $1, \alpha_{1}, \ldots, \alpha_{n}$.

Для доказательства результата в предположении условия Б надо действовать аналогично описанному вьше процессу и использовать тот факт, что по причине единственности касательной гиперплоскости к сфере $\partial B_{f}^{1}$ можно обеспечить трансверсальность пересечения прямой $l^{*}=\pi^{*} \cap\left\{x=p_{\nu}\right\}$ сферы $\partial B_{f}^{1}$.

8. Асимптотические направления. В этом пункте мы приведем без доказательств простые следствия из теорем 2, 3 и 4 в терминах асимптотических направлений для $f$-н.п.

Асимптотическим направлением для $f$-н.п. к набору $\alpha$ назьвается такая точка $\theta \in \partial B_{f}^{1}(0)$, для которой найдется подпоследовательность $\nu_{j}$ такая, что

$$
\lim _{j \rightarrow+\infty} \Xi_{\nu_{j}}=\theta
$$

Множество всех асимптотических направлений для $\alpha$ будем обозначать через $\Gamma_{f}(\alpha)$. Ясно, что $\Gamma_{f}(\alpha) \subseteq B_{f}^{1}(0)$ - замкнутое множество. Похожим, но несколько другим образом, асимптотические направления определяет Рождерс [15].

Множество $\mathscr{A} \subseteq B_{f}^{1}(0)$ будем называть $f$-асимптотически допустимы.м, если для

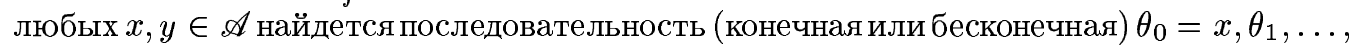
$\theta_{k}, \ldots, \theta_{j} \in \mathscr{A}$, такая, что

1) последовательность $\left\{\theta_{j}\right\}$ удовлетворяет условию А или условию Б из п. 7 ;

2) последний член последовательности равен $y$ или $\lim _{j \rightarrow \infty} \theta_{j}=y$.

Теорема 6. Если $\mathscr{A} \subseteq B_{f}^{1}(0)$ замкнуто и $f$-асимптотически допустимо, то найдется набор $\alpha \in \mathbb{R}^{n}$ чисел, линейно независимых вместе с единиией над $\mathbb{Z}$, таких, что $\mathscr{A}=\Gamma_{f}(\alpha)$.

СледствИЕ. Пусть норма $f$ гладкая. Если в замкнутом $\mathscr{A}$ найдется хотя бы одна пара иентрально симметричных относительно 0 точек, то найдется набор $\alpha \in \mathbb{R}^{n}$ чисел, линейно независимых вместе с единицей над $\mathbb{Z}$, таких, что $\mathscr{A}=\Gamma_{f}(\alpha)$.

Теорема 6 следует из теоремы 4. Она подтверждает наблюдение Роджерса [15] о том, что множество асимптотических направлений не обязано быть центрально симметричным, но тем не менее некоторое подобие симметрии у него имеет место. По-видимому, следствие верно и для многих негладких норм, например для нормы $\max _{j}\left|x_{j}\right|$. 
Из теоремы 2 вытекает

ТЕорема 7. Пусть норма $f$ строго выпукла. Тогда найдется полохительное $\delta_{1}$, зависящее от $f$, такое, что если множсество $\mathscr{A} \subset \partial B_{f}^{1}(0)$ таково, что

$$
\mathscr{A} \subset \operatorname{int} B_{f}^{1+\delta_{1}}(\theta) \quad \forall \theta \in \mathscr{A},
$$

то не сущестует вектора $\alpha \in \mathbb{R}^{n}$ такого, ито $\mathscr{A}=\Gamma_{f}(\alpha)$.

В заключение автор хочет отметить, что поводом к написанию настоящей работы послужила статья [16] (в которой дан обзор результатов о распределении наилучших приближений в свете теории возмущений уравнений Гамильтона) и общение с профессором Пьером Лошаком на конференщии в Йорке осенью 1997 года. Впервые автор докладывал результаты настоящей работы на конференции по теории чисел в Люмини весной 1998 года [17].

\section{СПИСОК ЦИТИРОВАННОЙ ЛИТЕРАТУРЫ}

[1] Касселс Дж. В. С. Введение в геометрию чисел. М.: Мир, 1965.

[2] Мощевитин Н. Г. О наилучших совместных приближениях // УМН. 1996. Т. 51. №6. C. $214-215$.

[3] Khinchin A. Ya. Über eine Klasse linearer Diophantischer Approximationen // Rend. Circ. Mat. Palermo. 1926. V. 50. P. 170-195.

[4] Хинчин А.Я. Регулярные системы линейных уравнений и общая задача Чебышева // Изв. АН ССCР. Сер. матем. 1948. Т. 12. №3. С. 249-258.

[5] Lagarias J.S. Best simultaneous Diophantine approximation. II // Pacific J. Math. 1982. V. 102. № 1. P. 61-88.

[6] Мощевитин Н. Г. О геометрии наилучших приближений // Докл. РАН. 1998. Т. 359. № 5. C. $587-589$.

[7] Мощевитин Н.Г. Многомерные диофантовы приближения и динамические системы // Регулярная и хаотическая динамика. 1997. Т. 2. №1. С. 81-95.

[8] Касселс Дж. В. С. Введение в теорию диофантовых приближений. М., 1961.

[9] Хинчин А.Я. Цепные дроби. М.: Наука, 1978.

[10] Rogers C. A. The signatures of the errors of simultaneous Diophantine approximations // Proc. London Math. Soc. Ser. 2. 1951. V. 52. P. 186-190.

[11] Cassels J. W. S. Simultaneous Diophantine approximations // Proc. London Math. Soc. (3). 1955. V. 5. P. 435-448.

[12] Мощевитин Н. Г. О совместных диофантовых приближениях // Матем. заметки. 1997. T. 61 . № 5. C. $706-716$.

[13] Lagarias J. S. Best simultaneous Diophantine approximation. I // Trans. Amer. Math. Soc. 1982. V. 272. № 2. P. 545-554.

[14] Sos V. T., Szekeres G. Rational approximation vectors // Acta Arith. 1988. V. 49. № 3. P. 255-261.

[15] Rogers C. A. The asymptotic directions of $n$ linear forms in $n+1$ integrall variables // Proc. London Math. Soc. Ser. 2. 1951. V. 52. P. 161-185.

[16] Лошак П. Каноническая теория возмущений: подход, основанный на совместных приближениях // УМН. 1992. Т. 47. №67. С. 59-140.

[17] Moshchevitin N. Best approximations: convex functions, signatures and asymptotic directions // Diophantine Approximations. Conference at CIRM (Luminy, 18-22 Mai, 1998). Abstracts of talks. 